PROCEEDINGS OF THE

AMERICAN MATHEMATICAL SOCIETY

Volume 128, Number 4, Pages 1135-1140

S 0002-9939(99)05434-9

Article electronically published on October 27, 1999

\title{
UNIONS OF JOHN DOMAINS
}

\author{
JUSSI VÄISÄLÄ \\ (Communicated by Christopher D. Sogge)
}

\begin{abstract}
We study the question: When is the union of John domains a John domain?
\end{abstract}

\section{INTRODUCTION}

1.1. John domains in the euclidean $n$-space $R^{n}$ were introduced by F. John [Jo] in connection with his work on elasticity. The term is due to O. Martio and J. Sarvas MS. Roughly speaking, a domain is a John domain if it is possible to travel from one point of the domain to another without going too close to the boundary.

Several equivalent characterizations for John domains can be found in [NV]. In the present paper we shall adopt the definition based on diameter cigars. Let $\gamma \subset R^{n}$ be an arc with endpoints $a$ and $b$; we shall write this as $\gamma: a \curvearrowright b$. For $x \in \gamma$ we set

$$
\zeta(x, \gamma)=d(\gamma[a, x]) \wedge d(\gamma[x, b])
$$

Here $\gamma[a, x]$ is the subarc of $\gamma$ between $a$ and $x, d()$ denotes diameter, and $r \wedge s=$ $\min (r, s)$. For $c \geq 1$, we say that $\gamma$ is a $c$-John arc in a domain $D \subset R^{n}$ if $\gamma \subset D$ and if

$$
\zeta(x, \gamma) \leq c d(x, \partial D)
$$

for all $x \in \gamma$, where $d($,$) denotes distance with d(x, \varnothing)=\infty$. A domain $D$ is a $c$-John domain if each pair of distinct points in $D$ can be joined by a $c$-John arc.

Suppose that $D_{1}$ and $D_{2}$ are $c$-John domains in $R^{n}$ with $D_{1} \cap D_{2} \neq \varnothing$. The union $D=D_{1} \cup D_{2}$ need not be a John domain. For example, let $H$ and $H^{\prime}$ be the upper and lower half spaces of $R^{n}$, respectively. Then $H$ and $H^{\prime} \cup B^{n}$ are John domains but their union is not. If one of the domains is bounded, then $D$ is a $c^{\prime}$-John domain for some $c^{\prime}$ by 3.1 or by 3.4 but $c^{\prime}$ can be arbitrarily large. In the main result 3.4 we show that if the volume of $D_{1} \cap D_{2}$ is comparable to the volume of one of the domains $D_{1}, D_{2}$, then we obtain an upper bound for $c^{\prime}$. In this result, one cannot replace volume by diameter, except for the special case where the domains are simply connected domains in the plane with connected intersection.

I thank Ritva Hurri-Syrjänen for calling my attention to this problem.

1.2. Notation. For $x \in R^{n}$ and $r>0$, we let $B(x, r)$ and $\bar{B}(x, r)$ denote the open and closed balls in $R^{n}$ with center $x$ and radius $r$, and we write $B(r)=$

Received by the editors June 1, 1998.

2000 Mathematics Subject Classification. Primary 30C65.

Key words and phrases. John domain. 
$B(0, r), \quad \bar{B}(r)=\bar{B}(0, r), B^{n}=B(0,1)$. For a measurable set $A \subset R^{n},|A|$ is the Lebesgue measure of $A$, and we set $\Omega_{n}=\left|B^{n}\right|$. Notation like $a b / x y z$ means $(a b) /(x y z)$.

\section{Preliminaries}

In this section we give various auxiliary results needed in the proof of the main result 3.4 .

2.1. Lemma. Let $A$ be a bounded set in $R^{n}$ and let $r>0$. Then there is a finite sequence of points $z_{1}, \ldots, z_{p}$ in $A$ such that the balls $B_{j}=B\left(z_{j}, r\right)$ cover $A$ and such that each point in $R^{n}$ belongs to at most $N_{n}$ balls $B_{j}$, where $N_{n}$ is a positive integer depending only on $n$.

Proof. Divide $R^{n}$ into a lattice of closed cubes of side $s=r / 2 \sqrt{n}$. For each cube $Q$ meeting $A$ choose a point $z_{Q} \in A \cap Q$. Since $Q \subset B\left(z_{Q}, r\right)$, the balls $B\left(z_{Q}, r\right)$ cover $A$. If the balls $B\left(z_{Q}, r\right)$ and $B\left(z_{R}, r\right)$ intersect, then $d(Q, R)<2 r=4 s \sqrt{n}$, and the lemma follows.

2.2. Plumpness. An open set $U \subset R^{n}$ is c-plump, $c \geq 1$, if for each $x \in U$ and $r>0$ with $U \backslash B(x, r) \neq \varnothing$, there is $z \in \bar{B}(x, r)$ with $B(z, r / c) \subset U$.

The following result is obvious:

2.3. Lemma. A c-John domain is 2c-plump. If $U$ is c-plump, then $\Omega_{n} d(U)^{n} \leq$ $c^{n}|U|$.

2.4. Notation. Let $U$ be an open proper subset of $R^{n}$. For $x \in U$ we write

$$
\delta(x)=d(x, \partial U) .
$$

For $t>0$ we set

$$
U(t)=\{x \in U: \delta(x)<t\}, \partial_{i} U(t)=\{x \in U: \delta(x)=t\} .
$$

Then $\partial U(t)=\partial U \cup \partial_{i} U(t)$. Since $\partial_{i} U(t)$ cannot have points of density, we get:

2.5. Lemma. $\left|\partial_{i} U(t)\right|=0$ for all $t>0$.

2.6. Lemma. If $U \subset R^{n}$ is open and c-plump and if $0<t<d(U) / 2 c$, then $\partial U \subset \partial_{i} U(t)+\bar{B}(c t)$.

Proof. Let $\varepsilon>0$. It suffices to show that $\partial U \subset \partial_{i} U(t)+B(c t+\varepsilon)$. Let $a \in \partial U$. Choose $x \in U$ with $|x-a|<\varepsilon$. Since $c t<d(U) / 2, U \backslash B(x, c t) \neq \varnothing$. Since $U$ is $c$-plump, there is $z \in \bar{B}(x, c t)$ with $\delta(z) \geq t$. Pick a point $y$ in the line segment $[a, z]$ with $\delta(y)=t$. Then $y \in \partial_{i} U(t)$, and $|a-y| \leq|a-z| \leq|a-x|+|x-z|<\varepsilon+c t$. Hence $a \in \partial_{i} U(t)+B(c t+\varepsilon)$.

2.7. Lemma. Suppose that $U \subset R^{n}$ is bounded, open and c-plump, and that $0<$ $t<d(U) / 2 c$. Then $|U(t)| \leq 8^{n} c^{n} N_{n}|V(t)|$, where $V(t)=\{x \in U: t / 2<\delta(x)<t\}$ and $N_{n}$ is given by 2.1.

Proof. The idea is from [MV (2.8)]. We remark that on the last line of p. 19 of MV], one should replace $B^{n}(x, t-s+c t)$ by $B^{n}(x, 2 t-s+c t)$.

Set $s=3 t / 4, A=\partial_{i} U(s)$. By 2.1 we can find a finite sequence of points $z_{1}, \ldots, z_{p}$ in $A$ such that the balls $B\left(z_{j}, t / 4\right)$ cover $A$ and such that each point in $R^{n}$ is covered at most $N_{n}$ times. 
By 2.6 we have $\partial U \subset A+\bar{B}(c s)$, and hence $U(t) \subset A+B(c s+t)$. Consequently, $U(t)$ is covered by the balls $B\left(z_{j}, t / 4+c s+t\right) \subset B\left(z_{j}, 2 c t\right), 1 \leq j \leq p$. Thus $|U(t)| \leq p \Omega_{n}(2 c t)^{n}$. On the other hand, since $B\left(z_{j}, t / 4\right) \subset V(t)$, we have $N_{n}|V(t)| \geq p \Omega_{n}(t / 4)^{n}$, and the lemma follows.

2.8. Theorem. Suppose that $U \subset R^{n}$ is open and c-plump with $d(U)=1$. Then $|U(t)| \leq \mu(t, c, n) \rightarrow 0$ as $t \rightarrow 0$.

Proof. Since $d(U)=1$, we have $|U(t)| \leq|U| \leq \Omega_{n}$ for all $t$. We may thus assume that $t \leq 1 / 3 c<d(U) / 2 c$. By 2.7 and 2.5 we obtain

$$
|U(t / 2)|=|U(t)|-|V(t)| \leq \lambda|U(t)|, \lambda=\lambda(c, n)=1-1 / 8^{n} c^{n} N_{n}<1 .
$$

Iterating this result gives $\left|U\left(2^{-k} t\right)\right| \leq \lambda^{k}|U(t)|$ for all positive integers $k$. Hence $\left|U\left(2^{-k} / 3 c\right)\right| \leq \lambda^{k} \Omega_{n}$ for all $k$, and the theorem follows.

2.9. Remark. The proof shows that one can choose $\mu(t, c, n)=\beta t^{\alpha}$ with $\alpha=$ $\alpha(c, n)>0$ and $\beta=\beta(n)>0$.

2.10. Corollary. Let $U$ be as in 2.8. Suppose that $A \subset U$ with $|A| \geq q>0$. Then there is $z \in A$ with $\delta(z) \geq r(c, q, n)>0$.

Proof. This holds with any $r$ such that $\mu(r, c, n)<q$.

\section{MAin Results}

3.1. Theorem. Suppose that $D_{1}$ and $D_{2}$ are c-John domains in $R^{n}$, and that $z \in$ $D_{1} \cap D_{2}$ and $r>0$ with $B(z, r) \subset D_{1} \cup D_{2}, d\left(D_{1}\right) \wedge d\left(D_{2}\right) \leq c_{0} r$. Then $D_{1} \cup D_{2}$ is a $c^{\prime}$-John domain with $c^{\prime}=2 c\left(c_{0}+1\right)$.

Proof. We may assume that $d\left(D_{1}\right)=1 \leq d\left(D_{2}\right)$. Then $r \geq 1 / c_{0}$. Let $a \in D_{1}, b \in$ $D_{2}$. Choose $c$-John $\operatorname{arcs} \alpha: a \curvearrowright z$ and $\beta: b \curvearrowright z$ in $D_{1}$ and $D_{2}$, respectively. The continuum $\alpha \cup \beta$ contains an arc $\gamma: a \curvearrowright b$. It suffices to show that $\gamma$ is a $c^{\prime}$-John arc in $D=D_{1} \cup D_{2}$.

For $x \in \alpha$ and $y \in \beta$ set

$$
\zeta_{1}(x)=d(\alpha[a, x]) \wedge d(\alpha[x, z] \cup \beta), \zeta_{2}(y)=d(\alpha \cup \beta[z, y]) \wedge d(\beta[y, b]) .
$$

It suffices to show that

$$
\zeta_{1}(x) \leq c^{\prime} d(x, \partial D)
$$

and

$$
\zeta_{2}(y) \leq c^{\prime} d(y, \partial D)
$$

for all $x \in \alpha$ and $y \in \beta$.

Choose a point $a_{1} \in \alpha$ dividing $\alpha$ to subarcs of equal diameter. Let $a_{2}$ be the last point of $\alpha$ with $\alpha\left[a_{2}, z\right] \subset \bar{B}(z, r / 2)$. Let $b_{1}$ and $b_{2}$ be the corresponding points in $\beta$.

Let $x \in \alpha$. To prove (3.2) we consider three cases.

Case 1. $x \in \alpha\left[a, a_{1}\right]$. Now $\zeta_{1}(x)=\zeta(x, \alpha) \leq c d\left(x, \partial D_{1}\right) \leq c d(x, \partial D)$.

Case 2. $x \in \alpha\left[a_{2}, z\right]$. Now $\zeta_{1}(x) \leq d(\alpha) \leq d\left(D_{1}\right)=1$ and $d(x, \partial D) \geq r / 2 \geq$ $1 / 2 c_{0}>1 / c^{\prime}$.

Case 3. $a_{2} \in \alpha\left[a_{1}, z\right]$ and $x \in \alpha\left[a_{1}, a_{2}\right]$. This case may be empty. We have $\zeta_{1}(x) \leq d\left(D_{1}\right)=1$, and $\zeta(x, \alpha) \geq r / 2$. Hence

$$
\zeta_{1}(x) \leq 2 \zeta(x, \alpha) / r \leq 2 c c_{0} d\left(x, \partial D_{1}\right) \leq 2 c c_{0} d(x, \partial D) .
$$


Next let $y \in \beta$. If $y \in \beta\left[b, b_{1}\right]$, we can argue as in Case 1 above. Suppose that $y \in \beta\left[b_{2}, z\right]$. Now $d(y, \partial D) \geq r / 2$, and

$$
\zeta_{2}(y) \leq d(\alpha \cup \beta[z, y]) \leq 1+r \leq 2\left(c_{0}+1\right) d(y, \partial D) .
$$

Finally, assume that $b_{2} \in \beta\left[b_{1}, z\right]$ and that $y \in \beta\left[b_{1}, b_{2}\right]$. This case may again be empty. Now

$$
\zeta_{2}(y) \leq d(\alpha)+d(\beta[z, y]) \leq 1+\zeta(y, \beta) \leq 1+c d\left(y, \partial D_{2}\right) \leq 1+c d(y, \partial D) .
$$

Since $r / 2 \leq \zeta(y, \beta) \leq c d(y, \partial D)$, we obtain $1 \leq 2 c c_{0} d(y, \partial D)$, and (3.3) follows.

3.4. Theorem. Suppose that $D_{1}, D_{2} \subset R^{n}$ are c-John domains with $\left|D_{1}\right| \wedge\left|D_{2}\right| \leq$ $c_{0}\left|D_{1} \cap D_{2}\right|<\infty$. Then $D_{1} \cup D_{2}$ is a $c^{\prime}$-John domain with $c^{\prime}=c^{\prime}\left(c, c_{0}, n\right)$.

Proof. By Lemma 2.3, the domains $D_{k}$ are 2c-plump, and at least one of them is bounded. Hence we may assume that $d\left(D_{1}\right)=1 \leq d\left(D_{2}\right)$. By 2.3 this gives $\left|D_{k}\right| \geq \Omega_{n} /(2 c)^{n}$, and hence $\left|D_{1} \cap D_{2}\right| \geq \Omega_{n} / c_{0}(2 c)^{n}$. By 2.10 we can find $z \in$ $D_{1} \cap D_{2}$ such that $d\left(z, \partial D_{1}\right) \geq r>0$, where $r$ depends only on $\left(c, c_{0}, n\right)$. Since $B(z, r) \subset D_{1} \subset D_{1} \cup D_{2}$, the theorem follows from 3.1

Theorem 3.4 can be applied to prove the John property of more general unions. As an example we give the following result:

3.5. Theorem. Suppose that $D_{0}, D_{1}, \ldots$ is a finite or infinite sequence of c-John domains in $R^{n}$. Suppose also that $\left|D_{j}\right| \leq c_{0}\left|D_{0} \cap D_{j}\right|$ for all $j \geq 1$. Then the domain $D=\bigcup\left\{D_{j}: j \geq 0\right\}$ is a $c_{1}$-John domain with $c_{1}=c_{1}\left(c, c_{0}, n\right)$.

Proof. Set $G_{j}=D_{0} \cup D_{j}$ for $j \geq 1$. Since $\left|D_{0}\right| \wedge\left|D_{j}\right| \leq\left|D_{j}\right| \leq c_{0}\left|D_{0} \cap D_{j}\right|, G_{j}$ is a $c^{\prime}$-John domain with $c^{\prime}=c^{\prime}\left(c_{0}, c, n\right) \geq c$ by 3.4

Assume that $1 \leq j<k$ and that $a \in D_{j}, b \in D_{k}$. It suffices to find $c_{1} \geq 1$ and a $c_{1}$-John $\operatorname{arc} \gamma: a \curvearrowright b$ in $D$. For this, it suffices to show that $G_{j} \cup D_{k}$ is a $c_{1}$-John domain. This follows from 3.4 since

$$
\left|G_{j}\right| \wedge\left|D_{k}\right| \leq\left|D_{k}\right| \leq c_{0}\left|D_{0} \cap D_{k}\right| \leq c_{0}\left|G_{j} \cap D_{k}\right|,
$$

and hence the theorem holds with $c_{1}=c^{\prime}\left(c^{\prime}\left(c, c_{0}, n\right), c_{0}, n\right)$.

3.6. Diameter conditions. One may ask whether the measure condition of 3.4 can be replaced by the diameter condition

$$
d\left(D_{1}\right) \wedge d\left(D_{2}\right) \leq c_{0} d\left(D_{1} \cap D_{2}\right)<\infty .
$$

It is easy to see, however, that in this case, $D=D_{1} \cup D_{2}$ need not be a John domain with any reasonable constant. An example where $V=D_{1} \cap D_{2}$ is connected is given by two cubes in $R^{3}$ which intersect in a narrow cylinder $V$. In $R^{2}$, an example with connected $V$ is not quite so simple. Let $Q$ be the open unit square $\{(x, y): 0<x<1,0<y<1\}$. Let $k$ be a positive integer, and set $D_{1}=$ $Q \backslash\left\{\left(2^{-k}, j 2^{-k}\right): 1 \leq j \leq 2^{k}-1\right\}$. Then $D_{1}$ is a $c$-John domain with $c$ independent of $k$. Let $D_{2}$ be the half plane $\left\{(x, y): x<2^{-k-1}\right\}$. Then $V=D_{1} \cap D_{2}$ is connected and $d(V)>1$, but the John constant of $D_{1} \cup D_{2}$ tends to $\infty$ as $k \rightarrow \infty$.

In the last example above, $D_{1}$ is not simply connected. We next show that for simply connected planar domains, the diameter condition gives an upper bound for the John constant of $D$. The proof makes use of some basic results in plane topology, which will be considered in Section 4 
3.7. Theorem. Suppose that $D_{1}$ and $D_{2}$ are simply connected $c$-John domains in $R^{2}$, that $D_{1} \cap D_{2}$ is connected, and that $d\left(D_{1}\right) \wedge d\left(D_{2}\right) \leq c_{0} d\left(D_{1} \cap D_{2}\right)<\infty$. Then $D_{1} \cup D_{2}$ is a $c^{\prime}$-John domain with $c^{\prime}=c^{\prime}\left(c, c_{0}\right)$.

Proof. Write $D=D_{1} \cup D_{2}, V=D_{1} \cap D_{2}$. We may assume that $d\left(D_{1}\right)=1 \leq d\left(D_{2}\right)$; then $d(V) \geq 1 / c_{0}$. Set $t=\left(16 c c_{0}\right)^{-1}$. If $d\left(x, \partial D_{k}\right) \geq t$ for some $x \in V$ and for some $k=1,2$, the theorem follows from 3.1 Hence we may assume that

$$
d\left(x, \partial D_{k}\right)<t
$$

for all $x \in V$ and for $k=1,2$.

Fix $a, b \in V$ with $|a-b|>1 / 2 c_{0}$. For $k=1,2$, choose a $c$-John arc $\alpha_{k}: a \curvearrowright b$ in $D_{k}$. If $x \in \alpha_{k}$ and $|x-a| \wedge|x-b| \geq c t$, then $d\left(x, \partial D_{k}\right) \geq t$, and hence $x \notin V$ by (3.8). Consequently,

$$
\alpha_{k} \cap V \subset B(a, c t) \cup B(b, c t)
$$

for $k=1,2$.

Since $2 c t=1 / 8 c_{0}<1 / 2 c_{0}<|a-b|$, the balls $B(a, c t)$ and $B(b, c t)$ are disjoint. Since $\alpha_{1} \cap \alpha_{2} \subset V$, it follows from (3.9) that there are points $a^{\prime} \in \alpha_{1} \cap \alpha_{2} \cap B(a, c t)$ and $b^{\prime} \in \alpha_{1} \cap \alpha_{2} \cap B(b, c t)$ such that the arcs $\alpha_{k}^{\prime}=\alpha_{k}\left[a^{\prime}, b^{\prime}\right]$ meet only at the common endpoints $a^{\prime}, b^{\prime}$. Thus $J=\alpha_{1}^{\prime} \cup \alpha_{2}^{\prime}$ is a Jordan curve in $D$. Since $D$ is simply connected by 4.2 we have $i(J) \subset D$.

Let $L$ be the line through $(a+b) / 2$, perpendicular to $[a, b]$. Then $L$ separates $a^{\prime}$ and $b^{\prime}$ in $R^{2}$. By 4.4 there are points $x_{k} \in \alpha_{k}^{\prime} \cap L$ such that the open line segment ]$x_{1}, x_{2}$ [ lies in $i(J)$. By (3.9) we have $x_{2} \notin D_{1}$ and $x_{1} \notin D_{2}$. Since $V$ is connected, we can choose an arc $\gamma: a^{\prime} \curvearrowright b^{\prime}$ in $V$. Since $\gamma$ and $\alpha_{1}^{\prime}$ lie in the simply connected domain $D_{1}$, we have $\gamma \sim \alpha_{1}^{\prime}$ in $R^{2} \backslash\left\{x_{2}\right\}$, and similarly $\gamma \sim \alpha_{2}^{\prime}$ in $R^{2} \backslash\left\{x_{1}\right\}$. Hence we can apply Lemma 4.3 with the substitution $\left(a, b, \alpha_{1}, \alpha_{2}, \beta\right) \mapsto\left(a^{\prime}, b^{\prime}, \alpha_{1}^{\prime}, \alpha_{2}^{\prime},\left[x_{1}, x_{2}\right]\right)$ to find a point $z \in \gamma \cap] x_{1}, x_{2}[\subset i(J)$.

It suffices to show that $d(z, J) \geq t$, because then $B(z, t) \subset D$, and the theorem follows from 3.1 Assume that $x \in J$ with $|x-z|<t$. Then

$$
|x-a| \geq|z-a|-|x-z|>|a-b| / 2-t>1 / 4 c_{0}-1 / 16 c c_{0}>1 / 8 c_{0},
$$

and similarly $|x-b|>1 / 8 c_{0}$. We may assume that $x \in \alpha_{1}$. Since $\alpha_{1}$ is a $c$-John arc in $D_{1}$, we have $d\left(x, \partial D_{1}\right)>1 / 8 c c_{0}=2 t$. Since $z \in \gamma \subset V$, (3.8) gives $d\left(z, \partial D_{1}\right)<t$, and we obtain the contradiction

$$
t>|z-x| \geq d\left(x, \partial D_{1}\right)-d\left(z, \partial D_{1}\right)>2 t-t=t .
$$

\section{Some topology of the Plane}

4.1. General remarks. The purpose of this section is to prove some basic results of plane topology, needed in the proof of 3.7 We recall that a domain $D \subset R^{2}$ is simply connected if and only if the singular homology group $H_{1}(D)$ is trivial, and also if and only if the complement of $D$ in the extended plane is connected.

Suppose that $A \subset R^{2}$ and that $\alpha_{1}, \alpha_{2}: a \curvearrowright b$ are $\operatorname{arcs}$ in $A$. Choose homeomorphisms $\alpha_{k}^{*}:[0,1] \rightarrow \alpha_{k}$ with $\alpha_{k}^{*}(0)=a, \alpha_{k}^{*}(1)=b, k=1,2$. If $\alpha_{1}^{*}$ is path homotopic to $\alpha_{2}^{*}$ in $A$, we say that $\alpha_{1}$ is homotopic to $\alpha_{2}$ in $A$ and write $\alpha_{1} \sim \alpha_{2}$ in $A$.

For an $\operatorname{arc} \alpha: a \curvearrowright b$, we let $\hat{\alpha}=\alpha \backslash\{a, b\}$ denote the set of its interior points. If $J \subset R^{2}$ is a Jordan curve, the bounded component of $R^{2} \backslash J$ is written as $i(J)$. 
4.2. Lemma. Suppose that $D_{1}$ and $D_{2}$ are simply connected domains in $R^{2}$ and that $D_{1} \cap D_{2}$ is nonempty and connected. Then $D_{1} \cup D_{2}$ is simply connected.

Proof. This is well known and follows from the exact Mayer-Vietoris sequence $0=$ $H_{1}\left(D_{1}\right) \oplus H_{1}\left(D_{2}\right) \rightarrow H_{1}\left(D_{1} \cup D_{2}\right) \rightarrow \tilde{H}_{0}\left(D_{1} \cap D_{2}\right)=0$.

4.3. Lemma. Suppose that $\alpha_{1}, \alpha_{2}: a \curvearrowright b$ are arcs in $R^{2}$ with $\alpha_{1} \cap \alpha_{2}=\{a, b\}$, and that $\beta: x_{1} \curvearrowright x_{2}$ is an arc with $x_{k} \in \hat{\alpha}_{k}$ and $\hat{\beta} \subset i\left(\alpha_{1} \cup \alpha_{2}\right)$. Suppose also that $\gamma: a \curvearrowright b$ is an arc in $R^{2} \backslash\left\{x_{1}, x_{2}\right\}$ such that $\gamma \sim \alpha_{1}$ in $R^{2} \backslash\left\{x_{2}\right\}$ and $\gamma \sim \alpha_{2}$ in $R^{2} \backslash\left\{x_{1}\right\}$. Then $\gamma$ meets $\beta$.

Proof. Assume that $\gamma \cap \beta=\varnothing$. Fix a point $z \in \hat{\beta}$. Since the inclusion $R^{2} \backslash \beta\left[z, x_{2}\right] \hookrightarrow$ $R^{2} \backslash\left\{x_{2}\right\}$ is a homotopy equivalence, we have $\gamma \sim \alpha_{1}$ in $R^{2} \backslash \beta\left[z, x_{2}\right]$, and hence in $R^{2} \backslash\{z\}$. Similarly, $\gamma \sim \alpha_{2}$ in $R^{2} \backslash\{z\}$, and hence $\alpha_{1} \sim \alpha_{2}$ in $R^{2} \backslash\{z\}$. Since $z \in i\left(\alpha_{1} \cup \alpha_{2}\right)$, this is well known to be a contradiction.

4.4. Lemma. Let $J \subset R^{2}$ be a Jordan curve, and let $a, b$ be points in $J$ dividing $J$ into subarcs $\alpha_{1}, \alpha_{2}$. Let $L \subset R^{2}$ be a line separating $a$ and $b$ in $R^{2}$. Then there are $x_{1} \in \alpha_{1} \cap L$ and $x_{2} \in \alpha_{2} \cap L$ such that $] x_{1}, x_{2}[\subset i(J)$.

Proof. Choose an endcut $\gamma_{a}$ of $D=i(J)$ with endpoints $a$ and $a^{\prime} \in D$ such that $\gamma_{a} \cap L=\varnothing$. Similarly choose an endcut $\gamma_{b}: b^{\prime} \curvearrowright b$. Since $L \cap D$ is closed in $D$ and separates the points $a^{\prime}$ and $b^{\prime}$ in $D$, there is a component $F$ of $L \cap D$ separating $a^{\prime}$ and $b^{\prime}$ in $D$; see [Ne, V.14.3]. Now $\left.F=\right] x_{1}, x_{2}\left[\right.$ with some $x_{1}, x_{2} \in \partial D$, and $\left[x_{1}, x_{2}\right]$ is a crosscut of $D$. From [Ne, V.11.8] it follows that $x_{1}$ and $x_{2}$ lie in different components of $J \backslash\{a, b\}$.

\section{REFERENCES}

[Jo] F. John, Rotation and strain, Comm. Pure. Appl. Math. 14 (1961), 391-413. MR 25:1672

[MS] O. Martio and J. Sarvas, Injectivity theorems in plane and space, Ann. Acad. Sci. Fenn. Math. 4 (1978), 383-401. MR 81i:30039

[MV] O. Martio and M. Vuorinen, Whitney cubes, p-capacity, and Minkowski content, Exposition. Math. 5 (1987), 17-40. MR 88e:28004

[NV] R. Näkki and J. Väisälä, John disks, Exposition. Math. 9 (1991), 3-43. MR 92i:30021

[Ne] M.H.A. Newman, Elements of topology of plane sets of points, Cambridge University Press, 1961.

Matematikan laitos, Helsingin yliopisto, PL 4, 00014 Helsinki, Finland

E-mail address: jussi.vaisala@helsinki.fi 